\title{
PROPER MOTIONS OF OB STARS IN BOTH HEMISPHERES
}

\author{
HARUO YASUDA \\ Tokyo Astronomical Observatory, Japan
}

\begin{abstract}
From a comparison between the rotational velocities derived from radial velocities and space motions of OB stars, large systematic errors of FK4 proper motions in the southern hemisphere are evaluated; these may be expected, from the known accuracy of the FK4. The error of adopted distance scale is also examined. It is suggested that meridian observations of OB stars should be extended to the southern hemisphere to further researches, not only on stellar kinematics, but also on the fundamental system.
\end{abstract}

